\title{
Outer Synchronization between Complex Networks with Nonlinear Time-Delay Characteristics and Time-Varying Topological Structures
}

\author{
Xinli Fang, Qiang Yang, and Wenjun Yan \\ College of Electrical Engineering, Zhejiang University, Hangzhou 310027, China \\ Correspondence should be addressed to Qiang Yang; qyang@zju.edu.cn
}

Received 6 December 2013; Accepted 15 February 2014; Published 25 March 2014

Academic Editor: Her-Terng Yau

Copyright (c) 2014 Xinli Fang et al. This is an open access article distributed under the Creative Commons Attribution License, which permits unrestricted use, distribution, and reproduction in any medium, provided the original work is properly cited.

This paper exploits the network outer synchronization problem in a generic context for complex networks with nonlinear time-delay characteristics and nonidentical time-varying topological structures. Based on the classic Lyapunov stability theory, the synchronization criteria and adaptive control strategy are presented, respectively, by adopting an appropriate LyapunovKrasovskii energy function and the convergence of the system error can also be well proved. The existing results of network outer synchronization can be obtained by giving certain conditions, for example, treating the coupling matrices as time-invariant, and by applying the suggested generic synchronization criteria and control scheme. The numerical simulation experiments for networks scenarios with dynamic chaotic characteristics and time-varying topologies are carried out and the result verifies the correctness and effectiveness of the proposed control solution.

\section{Introduction}

In essence, different from the network inner synchronization issue which considers the behaviors inside individual networks, the outer synchronization of dynamic complex networks looks into the collective behaviors between multiple networks. It is well known that various large-scale and complex networks need to work in a coordinated manner to fulfill their functionalities, for example, the power distribution network and underlying communication network for data acquisition and smart control or the communication system and its overlay control network for intelligent network resources management. Due to the fact that the networks can exhibit complex behaviors and dynamic nature, the synchronization between networks needs to be guaranteed in many cases to ensure that the collective behavior of multiple networks can meet the performance requirements. It has been virtually universally recognized that the outer synchronization problem and control strategies between multiple complex networks need to be well understood and investigated in order to well manage the networks for enhanced efficiency and performance [1-6].
In the literature, much research effort has been made to address the technical challenges from different aspects (e.g., [7-24]). The pioneer work was carried out in [7] and showed that the outer synchronization can be implemented between coupled unidirectional networks with identical topological structures. This inspired a collection of encouraging research findings and implementation attempts. In [8], the outer synchronization between two linear generalized complex networks is studied based on the Lyapunov stability theory, and a simple synchronization criterion for networks with the same topology was obtained. The authors in [9] designed and implemented an adaptive controller aiming to achieve the linear synchronization between two nonidentical dynamical networks. The aforementioned research outcome constrains to the linear outer synchronization, which consequently limits its performance for dynamic networks in practice. With such recognition, the outer synchronization between two delay-coupled networks with nonidentical topological structures and a noise perturbation was exploited and proved by adopting the stochastic LaSalle invariance principle in [10]. In [11], the authors presented a solution with scalar signal under pinning control for outer synchronization, and it 
highlighted that only a number of nodes need to be controlled and a few scalar signals were required by applying this control scheme. In [12], starting with a simple analysis on the synchronization error and eigenvalues of the matrices, the robustness of the outer synchronization is assessed for a range of network conditions and the result indicated that both for fractional-order and integer-order dynamics the proposed robust outer synchronization criteria are valid. Most recently, the criterion of generalized synchronization between two coupled networks was investigated in [13]. Also, the synchronization criteria of adaptive synchronization between two nonlinearly delay coupled complex networks with bidirectional interactions and nonidentical structures were studied in $[14,15]$. The hybrid synchronization problem between two networks with nondelayed and delayed coupling was addressed in $[16,17]$ and it demonstrated that the hybrid synchronization can be realized by adding adaptive pinning controller at a number of network nodes. In [18], the authors investigated the outer synchronization between uncertain complex delayed networks with adaptive coupling and a simple controller with update laws for the strengths of coupling configuration matrices was designed to realize the synchronization. The generalized function projective (lag, anticipated, and complete) synchronization between two networks was studied and a number of synchronization criteria were derived under various control strategies [1924]. It should be highlighted that almost all the existing results of network outer synchronization are obtained based on the strong assumption that the networks topologies and coupling strength do not vary over time. This implies that the available control solutions often cannot provide the expected synchronization performance for realistic networks whose topological structures and coupling strengths dynamically evolve.

To our best knowledge, current research effort on network outer synchronization considering time-varied network topologies mainly focused on the impact of varied coupling strength coefficient (e.g., [25-27]). In [28], the network synchronization with nonlinear oscillators through a local adaptive control approach and time-varying feedback coupling gains was studied, but it claimed that the suggested approach can only be effective under certain conditions which constrained its application scope. In [29], the authors looked into the synchronization problem between singular complex dynamical networks with time-varying delays and a condition for synchronization was derived based on the Linear Matrix Inequalities (LMI) theory. The synchronization problem for a class of complex networks where every identical node is a Lur'e system with time-varying delay was studied, and finally a delay-dependent synchronization criterion was derived and proved that it is less conservative by using LMI approach [30]. It should be noted that the work presented in $[29,30]$ only considered the node dynamics over time without taking the topological changes into account. In [31], the inner synchronization for networks with timedependent coupling and stochastically switching coupling structure based on the scrambling property was studied and sufficient conditions for a network to reach complete inner synchronization were reported. However, the technical challenge of synchronization criteria as well as the controller design to achieve outer synchronization for networks withnonlinear time-delay characteristics and time-varying topologies still remains as the existing research solutions cannot be directly applied. In this paper, we looked into this issue and attempt to address the technical challenge of network outer synchronization in a more generic manner, that is, between two networks with nonidentical time-varying topologies as well as nonlinear time-delay characteristics. The key technical contributions made in this paper can be summarized as follows: (1) the outer synchronization problem is investigated in a generic context for networks with nonidentical time-varying topological structures and the oscillators between networks are generalized with nonlinear time-delay characteristics; (2) through adopting an appropriate Lyapunov-Krasovskii energy function, we have proved the convergence of system errors and presented the controller design approach based on the suggested adaptive control strategy. As a result, the existing result of outer synchronization in current literature can be well derived from our proposed generic criteria once certain network conditions are given.

The remainder of this paper is organized as follows: the problem formulation and some preliminaries are described in Section 2; Section 3 presents the outer synchronization criteria along with proofs and a set of corollaries, followed by Section 4 which carries out the numerical study through an example to verify the proposed criteria and control solution; finally, Section 5 provides the conclusive remarks and points out the future research directions.

\section{Problem Formulations and Assumptions}

In this paper, we aim to investigate the outer synchronization issue in a generic condition, that is, between two nonlinear time-delay complex networks with inner coupling characteristics and nonidentical time-varying topological structures. It is assumed that the networks are with $N$ different dynamic oscillators and each oscillator inside the drive and response networks has $n$-dimensional states.

The drive dynamic complex network can be described as

$$
\begin{aligned}
\dot{x}_{i}(t)= & f\left(x_{i}(t)\right)+\sum_{j=1}^{N} a_{i j}(t) \cdot h\left(x_{j}(t)\right) \\
& +\sum_{j=1}^{N} a_{i j}^{\tau}(t) \cdot g\left(x_{j}(t-\tau)\right) .
\end{aligned}
$$

The response dynamic complex network is incorporated with an outer synchronization controller, and as a result, it can be described as the following expression:

$$
\begin{aligned}
\dot{y}_{i}(t)= & f\left(y_{i}(t)\right)+\sum_{j=1}^{N} b_{i j}(t) \cdot h\left(y_{j}(t)\right) \\
& +\sum_{j=1}^{N} b_{i j}^{\tau}(t) \cdot g\left(y_{j}(t-\tau)\right)+u_{i}(t),
\end{aligned}
$$


where $x_{i}(t)$ and $y_{i}(t)$ denote two $n$-dimensional state vectors in the drive and response networks, respectively; $\tau \geq 0$ is a time-delay constant between the drive and response networks; $f(\cdot): R^{n} \rightarrow R^{n}$ denotes a smooth function, which represents the self-dynamic functions of individual network nodes; $h(\cdot)$ and $g(\cdot)$ denote the nonlinear nondelay and timedelay inner activation functions, respectively, which describe the relationship of the current and the time-delay states of the $i$ th oscillator. $A(t)=\left(a_{i j}(t)\right)_{N \times N}\left(A^{\tau}(t), B(t)\right.$, and $\left.B^{\tau}(t)\right)$ are the time-varying topological coupling configuration matrices of the drive and response networks, respectively, which satisfy

$$
\begin{array}{ll}
a_{i i}(t)=-\sum_{j=1, j \neq i}^{N} a_{i j}(t), & a_{i i}^{\tau}(t)=-\sum_{j=1, j \neq i}^{N} a_{i j}^{\tau}(t), \\
b_{i i}(t)=-\sum_{j=1, j \neq i}^{N} b_{i j}(t), & b_{i i}^{\tau}(t)=-\sum_{j=1, j \neq i}^{N} b_{i j}^{\tau}(t) .
\end{array}
$$

$u_{i}(t): R^{n}(i=1, \ldots, N)$ is an $n$-dimension vector, which represents the control operations executed on the $i$ th response network oscillator.

Given a drive-response complex network system, the synchronization error of the two networks is defined as $e_{i}(t)=$ $y_{i}(t)-x_{i}(t)(i=1, \ldots, N)$ and from the expressions of drive network and response network, that is, (1) and (2); the dynamic error function can be written as the following form:

$$
\begin{aligned}
\dot{e}_{i}(t)= & {\left[f\left(y_{i}(t)\right)-f\left(x_{i}(t)\right)\right]+\sum_{j=1}^{N} b_{i j}(t) \cdot h\left(y_{j}(t)\right) } \\
& +\sum_{j=1}^{N} b_{i j}^{\tau}(t) \cdot g\left(y_{j}(t-\tau)\right)-\sum_{j=1}^{N} a_{i j}(t) \cdot h\left(x_{j}(t)\right) \\
& -\sum_{j=1}^{N} a_{i j}^{\tau}(t) \cdot g\left(x_{j}(t-\tau)\right)+u_{i}(t),
\end{aligned}
$$

where $i=1, \ldots, N$.

To implement the synchronization of the two networks under the control action, $u_{i}(t)$, when the time series $t$ goes to infinity, the system errors tend to be zero; that is, $\lim _{t \rightarrow \infty}\left\|e_{i}(t)\right\|=0$. Before we present the criteria of network outer synchronization, we start with a set of assumptions which are described as follows.

Assumption 1 (see [32]). It is assumed that there is a positive definite diagonal matrix, $Q=\operatorname{diag}\left(q_{1}, \ldots, q_{n}\right)$, and a diagonal matrix, $\Delta=\operatorname{diag}\left(\delta_{1}, \ldots, \delta_{n}\right)$, such that function $f(\cdot)$ satisfies the following inequality:

$$
\begin{gathered}
(\varepsilon-\eta)^{T} Q(f(\varepsilon, t)-f(\eta, t)-\Delta(\varepsilon-\eta)) \\
\leq-\mu(\varepsilon-\eta)^{T}(\varepsilon-\eta),
\end{gathered}
$$

where the constant $\mu \geq 0(\mu: R)$ and $t \geq 0$ is the system time series.
It is well known that many chaotic systems satisfy Assumption 1, for example, the Lorenz chaotic system, which will be described and used in our numerical simulation study.

Assumption 2 (see [33]). It is assumed that $h(\cdot)$ and $g(\cdot)$ are two Lipschitz continuousfunctions; that is, there exists two Lipschitz constants, $\alpha>0$ and $\beta>0$, such that

$$
\|h(\varepsilon)-h(\eta)\| \leq \alpha\|\varepsilon-\eta\|, \quad\|g(\varepsilon)-g(\eta)\| \leq \beta\|\varepsilon-\eta\|,
$$

where $\varepsilon, \eta \in R^{n}$.

This assumption ensures that the nonlinear nondelay and time-delay inner activation functions are two smooth functions and their changing rates, that is, the slope of the functions, are no more than a Lipschitz constant. This assumption is important in realistic complex networks as the evolving processes of the networks generally need to be guaranteed smooth by available methods and equipment to void adverse impacts to the network performance.

Assumption 3. In the time-varying topological coupling configuration matrices $A(t), A^{\tau}(t), B(t)$, and $B^{\tau}(t)$, the elements $a_{i j}(t), a_{i j}^{\tau}(t), b_{i j}(t)$, and $b_{i j}^{\tau}(t)$ are assumed to be bounded; that is, there exists the constants $a, a^{\tau}, b$, and $b^{\tau}$ such that $a_{i j}(t) \leq$ $a, a_{i j}^{\tau}(t) \leq a^{\tau}, b_{i j}(t) \leq b$, and $b_{i j}^{\tau}(t) \leq b^{\tau}$.

Remark 4. Assumption 3 confines to the actual network topological structure evolving features under any normal conditions; although the topological coupling matrices maybe time-varying, the coupling strengths of a network cannot be infinite; that is, they must be bounded.

Lemma 5 (Young's inequality [30]). For any vectors $x, y \notin R^{n}$, and any constant $c>0$, the following matrix inequality holds:

$$
x^{T} y \leq c x^{T} x+\frac{1}{4 c} y^{T} y .
$$

\section{Network Outer Synchronization: Criteria, Controller Design, and Error Convergence}

This section addresses the implementation of outer synchronization between the drive and response networks with timedelay characteristics and time-varying topological structures. By taking a theoretical approach based on the classic Lyapunov stability theory, we derive the criteria of network outer synchronization and present an adaptive control approach through introducing an appropriate Lyapunov-Krasovskii energy function and also prove the convergence of the system error.

Theorem 6. Suppose Assumptions 1-3 hold; the controller design

$$
\begin{aligned}
u_{i}(t)= & \sum_{j=1}^{N}\left[p_{i j}(t) \cdot h\left(y_{j}(t)\right)+q_{i j}(t) \cdot g\left(x_{j}(t-\tau)\right)\right] \\
& -d_{i} \cdot e_{i}(t)
\end{aligned}
$$


with the update laws

$$
\begin{aligned}
& \dot{p}_{i j}(t)=\dot{a}_{i j}(t)-\dot{b}_{i j}(t)-e_{i}^{T}(t) \cdot h\left(y_{j}(t)\right), \\
& \dot{q}_{i j}(t)=\dot{a}_{i j}^{\tau}(t)-\dot{b}_{i j}^{\tau}(t)-e_{i}^{T}(t) \cdot g\left(x_{j}(t-\tau)\right)
\end{aligned}
$$

can realize the outer synchronization between the drive network and response network defined in (1) and (2). $T$ is the transpose of a matrix; $p_{i j}$ and $q_{i j}$ are two $n \times 1$ vectors; $d_{i}$ is the feedback strength with proper dimension.

Proof. Choose the Lyapunov-Krasovskii energy function as follows:

$$
\begin{aligned}
V= & \frac{1}{2} \sum_{i=1}^{N} e_{i}^{T}(t) e_{i}(t)+\gamma \sum_{i=1}^{N} \int_{t-\tau}^{t} e_{i}^{T}(\theta) e_{i}(\theta) d \theta \\
& +\frac{1}{2} \sum_{i=1}^{N} \sum_{j=1}^{N}\left(b_{i j}(t)-a_{i j}(t)+p_{i j}(t)\right)^{2} \\
& +\frac{1}{2} \sum_{i=1}^{N} \sum_{j=1}^{N}\left(b_{i j}^{\tau}(t)-a_{i j}^{\tau}(t)+q_{i j}(t)\right)^{2}, \\
\dot{V}=\sum_{i=1}^{N} e_{i}^{T}(t)\left\{\left[f\left(y_{i}(t)\right)-f\left(x_{i}(t)\right)\right]+\sum_{j=1}^{N} b_{i j}(t) \cdot h\left(y_{j}(t)\right)\right. & \left.+\dot{b}_{i j}^{\tau}(t)-\dot{a}_{i j}^{\tau}(t)+\dot{q}_{i j}(t)\right] \cdot \\
& +\sum_{j=1}^{N} b_{i j}^{\tau}(t) \cdot g\left(y_{j}(t-\tau)\right) \\
& -\sum_{j=1}^{N} a_{i j}(t) \cdot h\left(x_{j}(t)\right) \\
& +\sum_{i=1}^{N} \sum_{j=1}^{N}\left[b_{i j}(t)-a_{i j}(t)+p_{i j}(t)\right] \\
& -\sum_{j=1}^{N} a_{i j}^{\tau}(t) \cdot g\left(x_{j}(t-\tau)\right) \\
+\sum_{j=1}^{N}\left[e_{i j}^{T}(t) e_{i j}(t)-\gamma \sum_{i=1}^{N} e_{i}^{T}(t-\tau) \cdot h\left(y_{j}(t)\right)\right. & \left.+\dot{p}_{i j}(t)\right] \\
& \left.\left.+q_{i j}(t) \cdot g\left(x_{j}(t-\tau)\right)\right]-d_{i} \cdot e_{i}(t)\right\}
\end{aligned}
$$

Given that $a_{i j}(t), a_{i j}^{\tau}(t), b_{i j}(t)$, and $b_{i j}^{\tau}(t)$ are time-varying scalars; then, we have

$$
\begin{aligned}
& \dot{V}=\sum_{i=1}^{N} e_{i}^{T}(t)\left[f\left(y_{i}(t)\right)-f\left(x_{i}(t)\right)\right] \\
& +\sum_{i=1}^{N} \sum_{j=1}^{N} e_{i}^{T}(t) b_{i j}(t) h\left(y_{j}(t)\right) \\
& +\sum_{i=1}^{N} \sum_{j=1}^{N} e_{i}^{T}(t) b_{i j}^{\tau}(t) g\left(y_{j}(t-\tau)\right) \\
& -\sum_{i=1}^{N} \sum_{j=1}^{N} e_{i}^{T}(t) a_{i j}(t) h\left(x_{j}(t)\right) \\
& -\sum_{i=1}^{N} \sum_{j=1}^{N} e_{i}^{T}(t) a_{i j}^{\tau}(t) g\left(x_{j}(t-\tau)\right) \\
& +\sum_{i=1}^{N} \sum_{j=1}^{N} e_{i}^{T}(t) p_{i j}(t) h\left(y_{j}(t)\right) \\
& +\sum_{i=1}^{N} \sum_{j=1}^{N} e_{i}^{T}(t) q_{i j}(t) g\left(x_{j}(t-\tau)\right)-\sum_{i=1}^{N} e_{i}^{T}(t) \cdot d_{i} \cdot e(t) \\
& +\gamma \sum_{i=1}^{N} e_{i}^{T}(t) e_{i}(t)-\gamma \sum_{i=1}^{N} e_{i}^{T}(t-\tau) e_{i}(t-\tau) \\
& -\sum_{i=1}^{N} \sum_{j=1}^{N} b_{i j}(t) e_{i}^{T}(t) h\left(y_{j}(t)\right) \\
& +\sum_{i=1}^{N} \sum_{j=1}^{N} a_{i j}(t) e_{i}^{T}(t) h\left(y_{j}(t)\right) \\
& -\sum_{i=1}^{N} \sum_{j=1}^{N} p_{i j}(t) e_{i}^{T}(t) h\left(y_{j}(t)\right) \\
& -\sum_{i=1}^{N} \sum_{j=1}^{N} b_{i j}^{\tau}(t) e_{i}^{T}(t) g\left(x_{j}(t-\tau)\right) \\
& +\sum_{i=1}^{N} \sum_{j=1}^{N} a_{i j}^{\tau}(t) e_{i}^{T}(t) g\left(x_{j}(t-\tau)\right) \\
& -\sum_{i=1}^{N} \sum_{j=1}^{N} q_{i j}(t) e_{i}^{T}(t) g\left(x_{j}(t-\tau)\right) \\
& =\sum_{i=1}^{N} e_{i}^{T}(t)\left[f\left(y_{i}(t)\right)-f\left(x_{i}(t)\right)\right]-\sum_{i=1}^{N} e_{i}^{T}(t) \cdot d_{i} \cdot e(t) \\
& +\gamma \sum_{i=1}^{N} e_{i}^{T}(t) e_{i}(t)-\gamma \sum_{i=1}^{N} e_{i}^{T}(t-\tau) e_{i}(t-\tau)
\end{aligned}
$$




$$
\begin{aligned}
& +\sum_{i=1}^{N} \sum_{j=1}^{N} a_{i j}(t) e_{i}^{T}(t)\left[h\left(y_{j}(t)\right)-h\left(x_{j}(t)\right)\right] \\
& +\sum_{i=1}^{N} \sum_{j=1}^{N} b_{i j}^{\tau}(t) e_{i}^{T}(t)\left[g\left(y_{j}(t-\tau)\right)-g\left(x_{j}(t-\tau)\right)\right] \\
\leq & -\sum_{i=1}^{N} e_{i}^{T}(t) \cdot u_{i} \cdot e_{i}(t)+\sum_{i=1}^{N} e_{i}^{T}(t) \cdot \delta_{i} \cdot e_{i}(t) \\
& -\sum_{i=1}^{N} e_{i}^{T}(t) \cdot d_{i} \cdot e_{i}(t)+\gamma \sum_{i=1}^{N} e_{i}^{T}(t) e_{i}(t) \\
& -\gamma \sum_{i=1}^{N} e_{i}^{T}(t-\tau) e_{i}(t-\tau)+\sum_{i=1}^{N} \sum_{j=1}^{N} a_{i j}(t) \cdot e_{i}^{T}(t) \cdot \alpha_{j} \cdot e_{j}(t) \\
& +\sum_{i=1}^{N} \sum_{j=1}^{N} b_{i j}^{\tau}(t) \cdot e_{i}^{T}(t) \cdot \beta_{j} \cdot e_{j}(t) .
\end{aligned}
$$

Considering that for all $\alpha_{j}$ and for all $\beta_{j}$, there exists $\alpha_{j} \leq$ $\max _{1 \leq j \leq N}\left(\alpha_{j}\right)$ and $\beta_{j} \leq \max _{1 \leq j \leq N}\left(\beta_{j}\right)$; then, we define $\alpha_{\max } \equiv$ $\max _{1 \leq j \leq N}\left(\alpha_{j}\right)$ and $\beta_{\max } \equiv \max _{1 \leq j \leq N}\left(\beta_{j}\right)$. With Lemma 5 and Assumption 3, we can have the following:

$$
\begin{aligned}
& \dot{V} \leq-\sum_{i=1}^{N} e_{i}^{T}(t) \cdot u_{i} \cdot e_{i}(t)+\sum_{i=1}^{N} e_{i}^{T}(t) \cdot \delta_{i} \cdot e_{i}(t) \\
& -\sum_{i=1}^{N} e_{i}^{T}(t) \cdot d_{i} \cdot e_{i}(t)+\gamma \sum_{i=1}^{N} e_{i}^{T}(t) e_{i}(t) \\
& -\gamma \sum_{i=1}^{N} e_{i}^{T}(t-\tau) e_{i}(t-\tau) \\
& +a \cdot \alpha_{\max } \cdot\left[\frac{1}{2} \sum_{i=1}^{N} e_{i}^{T}(t) e_{i}(t)+\frac{1}{2} \sum_{j=1}^{N} e_{j}^{T}(t) e_{j}(t)\right] \\
& +b^{\tau} \cdot \beta_{\max } \cdot\left[\frac{1}{2} \sum_{i=1}^{N} e_{i}^{T}(t) e_{i}(t)+\frac{1}{2} \sum_{j=1}^{N} e_{j}^{T}(t-\tau) e_{j}(t-\tau)\right]
\end{aligned}
$$

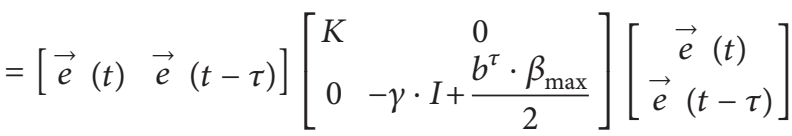

$$
\begin{aligned}
& =\left[\begin{array}{lll}
\vec{e} & (t) & \vec{e} \\
(t-\tau)
\end{array}\right] \Gamma\left[\begin{array}{c}
\vec{e}(t) \\
\vec{e}(t-\tau)
\end{array}\right],
\end{aligned}
$$

where

$$
\begin{aligned}
K & =-U+\Delta-D+\left(\gamma \cdot I+a \cdot \alpha_{\max }+\frac{b^{\tau} \cdot \beta}{2}\right) \otimes I, \\
\vec{e}(t) & =\left[e_{1}(t), e_{2}(t), \ldots, e_{N}(t)\right]_{n \times N^{\prime}} \\
\vec{e}(t-\tau) & =\left[e_{1}(t-\tau), e_{2}(t-\tau), \ldots, e_{N}(t-\tau)\right]_{n \times N^{*}}
\end{aligned}
$$

$U=\operatorname{diag}\left(u_{i}\right), \Delta=\operatorname{diag}\left(\delta_{i}\right)$, and $D=\operatorname{diag}\left(d_{i}\right) i=1,2, \ldots$, $N ; \otimes$ denotes Kronecher product; $I$ is an identity matrix.

From Assumption 3, it can be known that $a_{i j}(t), a_{i j}^{\tau}(t)$, $b_{i j}(t)$, and $b_{i j}^{\tau}(t)$ are bounded, and hence when the chosen constant, $\gamma$, and matrix, $D$, are large enough, then $\Gamma$ can be easily proved to be a negative definite matrix and the synchronization errors can be ensured to converge.

Remark 7. To prove $\Gamma$ to be a negative definite matrix, the minimum constant $\gamma$ can be derived as long as it satisfies $\gamma \cdot I \geq\left(b^{\tau} \cdot \beta_{\max }\right) / 2$. In addition, a larger feedback matrix, $D$, can lead to a faster convergence of errors during the process of network outer synchronization.

Remark 8. From the aforementioned proof of Theorem 6, it can be observed that if the elements of time-varying matrices, $A^{\tau}(t)$ and $B^{\tau}(t)$, are time-delay; that is, $t=t-\tau$, the outer synchronization criterion still holds.

Corollary 9. If the nonlinear inner activation functions, $h(\cdot)$ and $g(\cdot)$, are linear, that is,

$$
\begin{aligned}
\dot{x}_{i}(t)= & f\left(x_{i}(t)\right)+\sum_{j=1}^{N} a_{i j}(t) \cdot H \cdot x_{j}(t) \\
& +\sum_{j=1}^{N} a_{i j}^{\tau}(t) \cdot G \cdot x_{j}(t-\tau), \\
\dot{y}_{i}(t)= & f\left(y_{i}(t)\right)+\sum_{j=1}^{N} b_{i j}(t) \cdot H \cdot y_{j}(t) \\
& +\sum_{j=1}^{N} b_{i j}^{\tau}(t) \cdot G \cdot y_{j}(t-\tau)+u_{i}(t),
\end{aligned}
$$

where $h(\cdot)=H$ and $g(\cdot)=G$, then if we choose the controller as follows:

$$
\begin{aligned}
u_{i}(t)= & \sum_{j=1}^{N}\left[p_{i j}(t) \cdot H \cdot y_{j}(t)+q_{i j}(t) \cdot G \cdot x_{j}(t-\tau)\right] \\
& -d_{i} \cdot e_{i}(t)
\end{aligned}
$$

and the update laws as

$$
\begin{aligned}
& \dot{p}_{i j}(t)=\dot{a}_{i j}(t)-\dot{b}_{i j}(t)-e_{i}^{T}(t) \cdot H \cdot y_{j}(t), \\
& \dot{q}_{i j}(t)=\dot{a}_{i j}^{\tau}(t)-\dot{b}_{i j}^{\tau}(t)-e_{i}^{T}(t) \cdot G \cdot x_{j}(t-\tau),
\end{aligned}
$$


then the outer synchronization between networks (15) and (16) can be realized.

Corollary 10. If the network topological matrices $A(t), A^{\tau}(t)$, $B(t)$, and $B^{\tau}(t)$ are time-invariant, that is,

$$
\begin{aligned}
\dot{x}_{i}(t)= & f\left(x_{i}(t)\right)+\sum_{j=1}^{N} a_{i j} \cdot h\left(x_{j}(t)\right) \\
& +\sum_{j=1}^{N} a_{i j}^{\tau} \cdot g\left(x_{j}(t-\tau)\right) \\
\dot{y}_{i}(t)= & f\left(y_{i}(t)\right)+\sum_{j=1}^{N} b_{i j} \cdot h\left(y_{j}(t)\right) \\
& +\sum_{j=1}^{N} b_{i j}^{\tau} \cdot g\left(y_{j}(t-\tau)\right)+u_{i}(t),
\end{aligned}
$$

where $A(t)=A, A^{\tau}(t)=A^{\tau}, B(t)=B$, and $B^{\tau}(t)=B^{\tau}$, then if we choose the controller design as follows:

$$
\begin{aligned}
u_{i}(t)= & \sum_{j=1}^{N}\left[p_{i j}(t) \cdot H \cdot y_{j}(t)+q_{i j}(t) \cdot G \cdot x_{j}(t-\tau)\right] \\
& -d_{i} \cdot e_{i}(t)
\end{aligned}
$$

and the update laws as

$$
\begin{aligned}
& \dot{p}_{i j}(t)=-e_{i}^{T}(t) \cdot h\left(y_{j}(t)\right), \\
& \dot{q}_{i j}(t)=-e_{i}^{T}(t) \cdot g\left(x_{j}(t-\tau)\right),
\end{aligned}
$$

then the outer synchronization between networks (20) and (21) can be realized.

It can be seen that the obtained Corollaries 9 and 10 describe the outer synchronization between networks under linear or time-invariant scenarios, respectively. This makes the derived criteria of network outer synchronization more generic.The proofs of Corollaries 9 and 10 are similar to the proof of Theorem 6 and are not presented here due to the limitation of space.

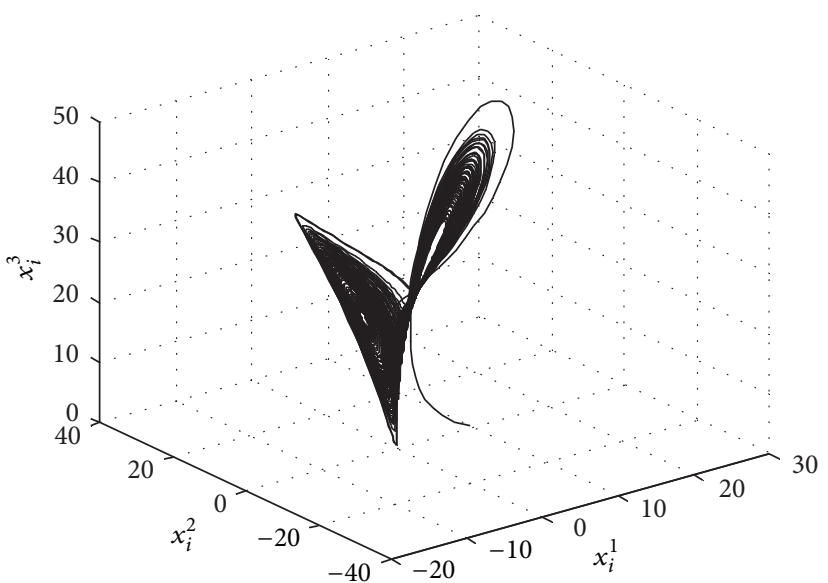

FIGURE 1: The chaotic characteristics of oscillation between the drive and response networks (with 5000 iterative steps and $i=1$ ).

\section{Numerical Simulation and Result}

In this section, we carry out a set of numerical simulation experiments by using a chaotic system as a case study to verify the effectiveness of the presented criteria of outer synchronization between networks with nonlinear timedelay characteristics and time-varying topologies. It is well known that the Lorenz system is a typical chaotic system, which can be described as follows:

$$
\dot{x}_{i}=\left\{\begin{array}{l}
10\left(x_{i}^{2}-x_{i}^{1}\right), \\
28 x_{i}^{1}-x_{i}^{2}-x_{i}^{1} x_{i}^{3}, \\
-\frac{\left(\alpha_{0}+8\right)}{3 x_{i}^{3}}+x_{i}^{1} x_{i}^{2},
\end{array}\right.
$$

where $\dot{x}_{i}=\left(\dot{x}_{i}^{1}, \dot{x}_{i}^{2}, \dot{x}_{i}^{3}\right)^{T}$, which stands for the three states of the $i$ th variable. When $\alpha_{0} \in[0,1]$, the above system can be guaranteed to be chaotic as illustrated in Figure 1, which shows the three states of a single node (e.g., $i=1$ ). In our simulation, for the sake of simplicity, we choose $\alpha_{0}=0$ and the time-delay constant $\tau=1$.

In our work, we choose the nonlinear inner coupling activation functions as $h(x)=0.5 \sin x$ and $g(y)=$ $-0.5 \cos y$, which meets Assumption 2. We assume that the drive and response networks have four nodes, and to ensure the generality of the experiment the initial states of the drive network and response network are configured with random values in the range of $(0,1)$ and $(0,10)$, respectively. The timevarying outer coupling topological matrices are derived from [27], which are expressed as follows:

$$
\begin{aligned}
& A=\left[\begin{array}{cccc}
2 \cos (t)-1 & \tanh (t) & -2 \cos (t) & 1-\tanh (t) \\
\sin (t) & -1-\sin (t) & \sin ^{2}(t) & \cos ^{2}(t) \\
\tanh (t)-\cos (t) & \sin (t) & -\tanh (t)+\cos (t) & -\sin (t) \\
\sin (t-3) & \sin (2 t) & -0.5 \sin (2 t)+\sin (3-t) & -0.5 \sin (2 t)
\end{array}\right], \\
& B=\left[\begin{array}{cccc}
-\cos ^{2}(t) & \cos (3 t) & 1-\cos (3 t) & -\sin ^{2}(t) \\
\sec (2 t) & \tanh (t) & -\sec (2 t) & -\tanh (t) \\
-0.5 \sin (2 t) & 1+\sin (2 t) & -\cos ^{2}(t) & -\sin ^{2}(t)-0.5 \sin (2 t) \\
\cos (t) & -\cos (t)+\tanh (t) & \sec (t)-\tanh (t) & -\sec (t)
\end{array}\right],
\end{aligned}
$$




$$
\begin{aligned}
A^{\tau}= & {\left[\begin{array}{cccc}
\cos (t) & \tanh (t) & -\cos (t) & -\tanh (t) \\
1-\tanh (t) & \cos (t) & -\cos (t) & \tanh (t)-1 \\
\sec (t)-\sin (t) & \sin (t)+\cos (t) & -\sec (t) & -\cos (t) \\
0.5 \sin (t) & -2 \tanh (t) & 2 \tanh (t) & -0.5 \sin (t)
\end{array}\right], } \\
B^{\tau}= & {\left[\begin{array}{cccc}
\sin (t+2) & \tanh ^{2}(t) & -\sin (t+2) & -\tanh ^{2}(t) \\
2 \sin (t) & -2 \sin (t) & \cos ^{2}(t) & -\cos ^{2}(t) \\
\cos (t)-1 & \sin ^{2}(t) & \cos ^{2}(t) & -\cos ^{2}(t) \\
1-\sin (t) & \sin (t) & -\sin ^{2}(t) & -\cos ^{2}(t)
\end{array}\right] . }
\end{aligned}
$$

Based on the aforementioned assumptions and experimental parameters, the dynamic states and errors of the driveresponse networks with the time-varying topological structures and nonlinear time-delay inner coupling activation functions are simulated by the use of Matlab version 2010, and the results are given in Figures 2(a)-2(d). It demonstrates the three nodes states of the drive network and corresponding response network and their synchronization errors against the simulation time respectively, where $x_{i}$ stands for the three states $\left(x_{i}^{1}, x_{i}^{2}\right.$, and $\left.x_{i}^{3}\right)$ of the $i$ th node in drive network and $y_{i}$ stands for the corresponding three states in the response network. $e_{i}$ is the synchronization error between $x_{i}$ and $y_{i}$. It can be seen that the node states of drive network and response network as well as the convergence errors exhibit chaos characteristics when no control actions are taken.

Figure 3 shows the network synchronization errors of each node in the drive and response networks when the control action is applied based on Theorem 6. Figures 3(a)3(d) demonstrate the impact of suggested control solution on the convergence of synchronization errors $e_{1}, e_{2}, e_{3}$, and $e_{4}$. It can be clearly seen that the states of errors gradually become less chaos and are pinned to the steady status, which confirms that the designed controller along with the update laws given in Theorem 6 can lead to the outer synchronization between the drive and response networks.

Through comparing the result presented in Figures 2(a)2(d) and 3(a)-3(d), the effectiveness of the proposed outer synchronization criteria can be clearly observed. Due to the chaos characteristics of the drive and response networks dynamics, the states error between the system nodes are chaotic, which are shown in Figures 2(a)-2(d). It can be seen that the error ranges are from 0 to $50(a, b, d)$ or even 0 to 100 (c). This indicates that the nonlinear time-delay driveresponse system can barely reach the outer synchronization between two networks with time-varying topological network structures by their autonomic dynamic process. Conversely, when the proposed adaptive control strategy is implemented and integrated into the experimented system, as shown in Figures 3(a)-3(d), the outer synchronization errors can be constrained within a small range (less than 4) and finally be converged to zero. This clearly indicates that the response network nodes can synchronize with the drive network nodes; that is, the system can effectively realize outer synchronization based on the proposed control approach. Though comparing the result presented in Figures 2 and 3, the effectiveness of the proposed outer synchronization strategy is verified, and the design of controller can be implemented.

Finally, to clearly observe the control impacts of the proposed control scheme, the dynamics process of the simulated controller is illustrated in Figure 4. Additionally, the relationship between the system errors and the adaptive control effects can be easily comprehended from Figure 4. It presents the dynamic of the convergence evolving process when the proposed design of controller, $u_{i}$, is applied in the network outer synchronization. With the implementation of outer synchronization between the drive and response networks, the system errors tend to be zero, which matches the theoretical analysis of (8) to (10); hence, according to its adaptive characteristics, the control effects are also gradually evolving to zero, and the main control effects time is about $10 \mathrm{~s}$, as shown in the following illustration. To better display the control range of the controller, the evolving process of the first 5 seconds is showed in detail in the small window of Figure 4 . From this picture, it can be seen that during the entire process, the control range is between \pm 5 , which is considered acceptable in engineering practice.

\section{Conclusive Remarks and Future Work}

The mechanisms and control strategy for network outer synchronization between complex networks are considered challenging and exploited for years. However, the existing control solutions are mostly obtained from the network scenarios with linear inner coupling connections and timeinvariant topological structures, which limit the applications in engineering practice. Unlike the existing studies, this paper looks into this issue in a generic context to address the network outer synchronization between complex networks with nonlinear time-delay characteristics and nonidentical time-varying topological structures. Based on the classic Lyapunov stability theory, we derived the criteria of outer synchronization and presented adaptive controller design by adopting an appropriate Lyapunov-Krasovskii energy function. The convergence of errors during network synchronization and the impact of control solution are well validated and evaluated through the numerical experiment for a generic network scenario. It should be highlighted 

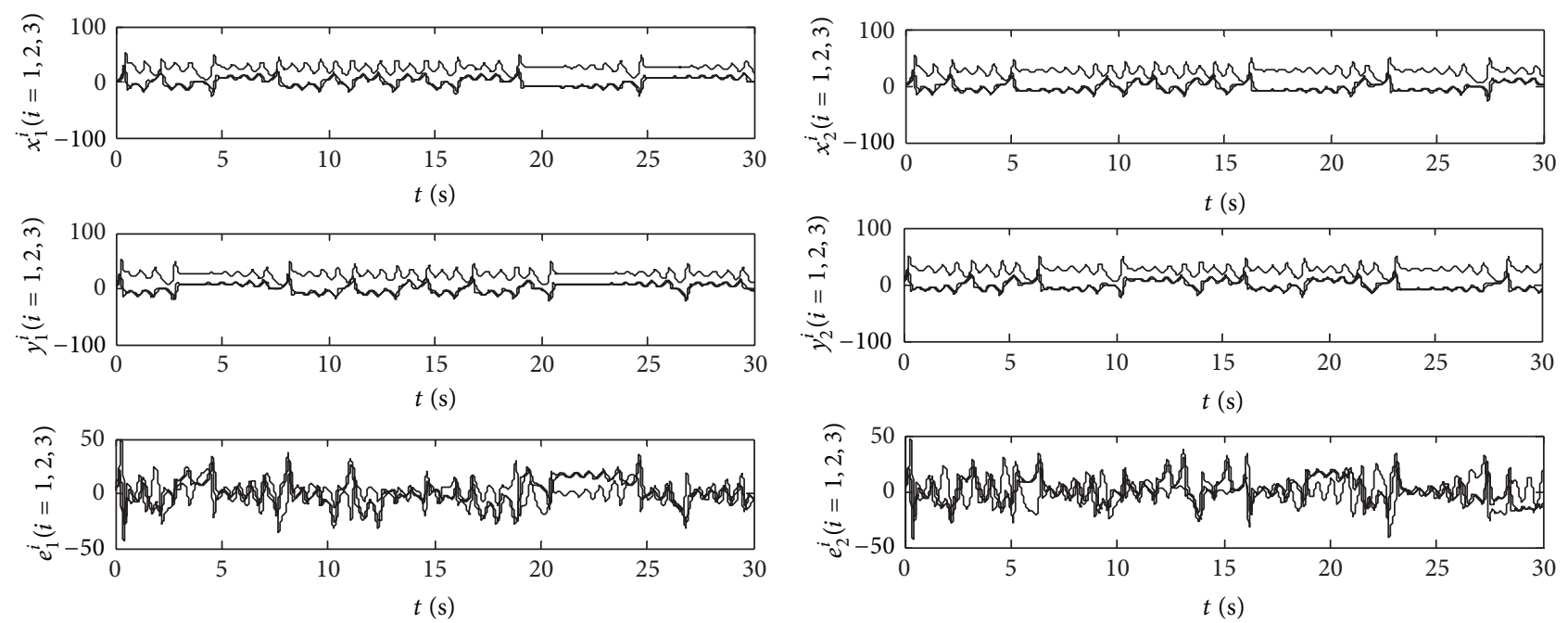

(a)

(b)
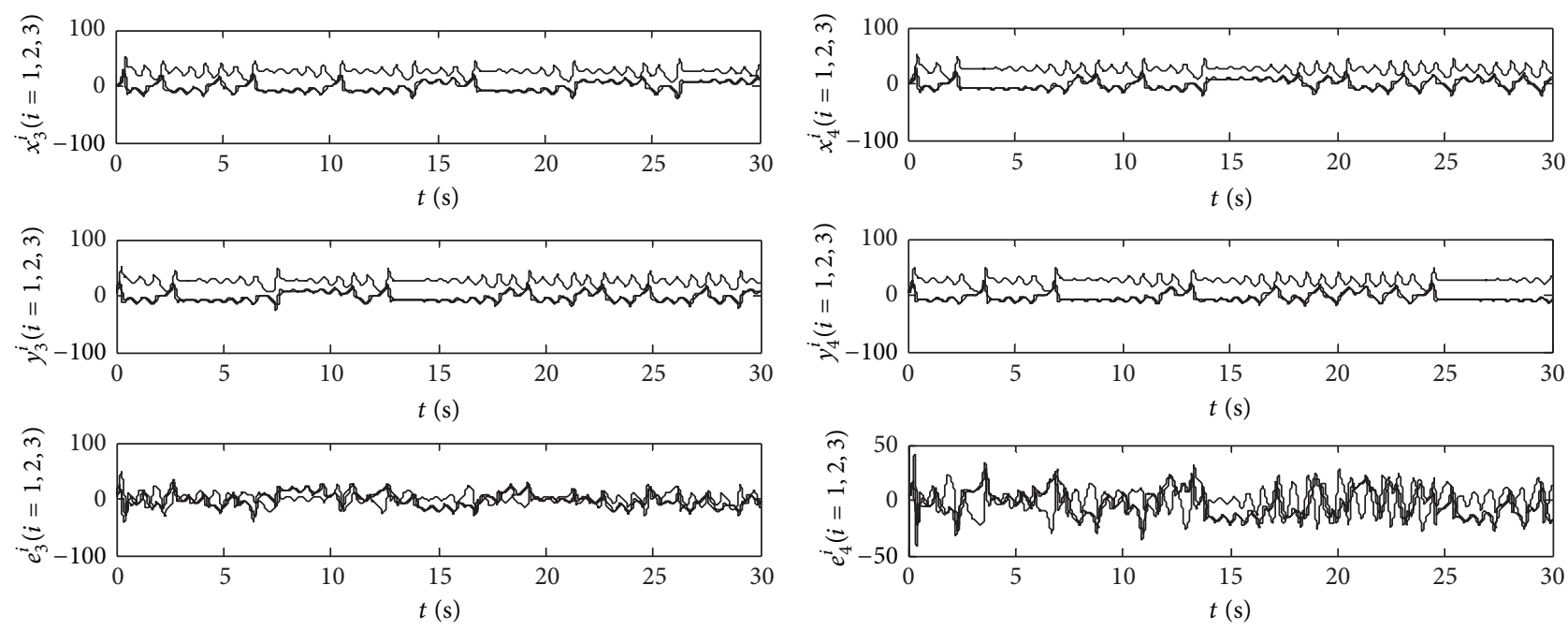

(c)

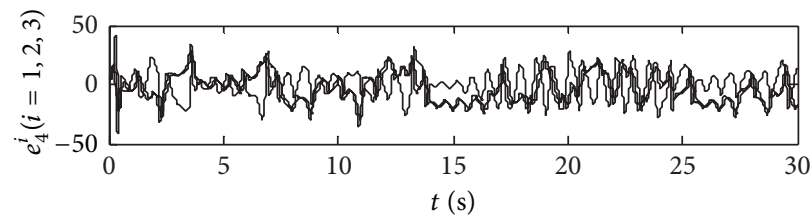

(d)

FIGURE 2: The states and synchronization errors evaluating without controls.

that the existing results of network outer synchronization in the literature can be effectively derived by giving certain conditions, for example, treating the coupling matrices as time-invariant, from the suggested generic synchronization criteria and control solution in the frame work.

In the analysis and simulation study in this work, we fully considered the impact of the time element to the outer synchronization of the drive-response network systems, including the nonlinear time-delay characteristics and the time-varying topological coupling configurations. In order to obtain a generic solution of synchronization criteria and control scheme, we neglected the particularities of networks. In fact, the dynamic processes of different oscillators are not always unified; as a result their dynamic characteristics under time-delay and time-varying topological scenarios need to be further investigated. Also we did not consider the environment factors, for example, noise, on the networks, which often affects the outer synchronization process of the drive-response network system. When the noise condition is presented, the control solution to be applied to realize the outer synchronization is still an outstanding challenge in the industry.

Therefore, in respect to the future work, we will further consider the outer synchronization problems between the networks with different dynamics oscillators under timeinvariant topological structures. Simultaneously, other environmental factors, for example, the noise, will be taken into account into the study to further improve the robustness of the control solutions.

\section{Conflict of Interests}

The authors declare that there is no conflict of interests regarding the publication of this paper. 


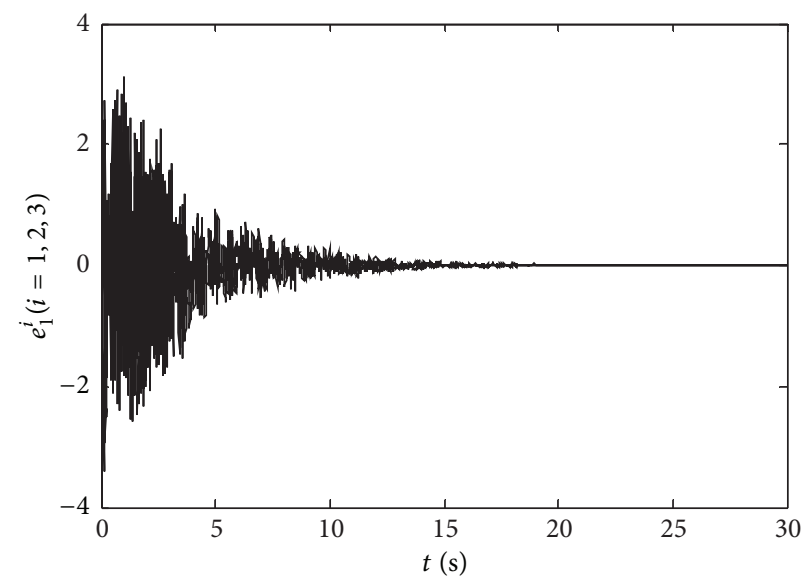

(a)

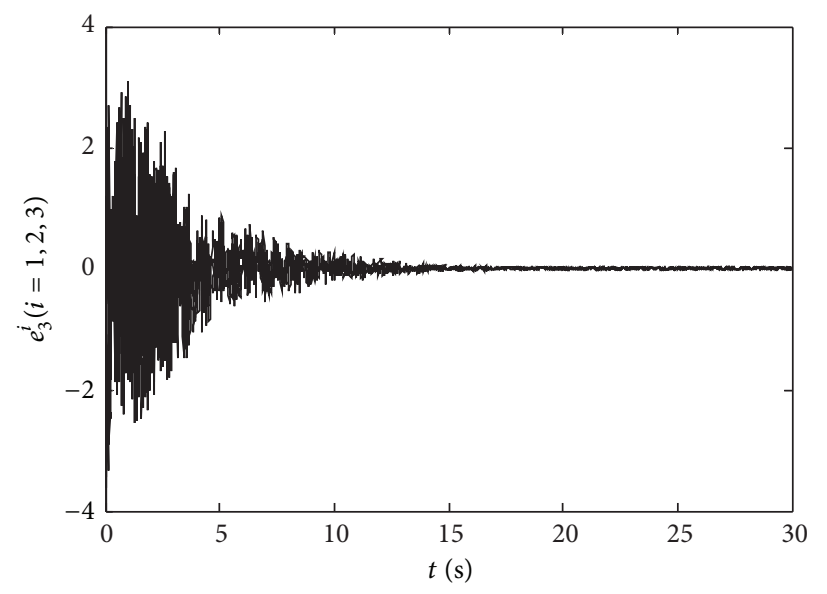

(c)

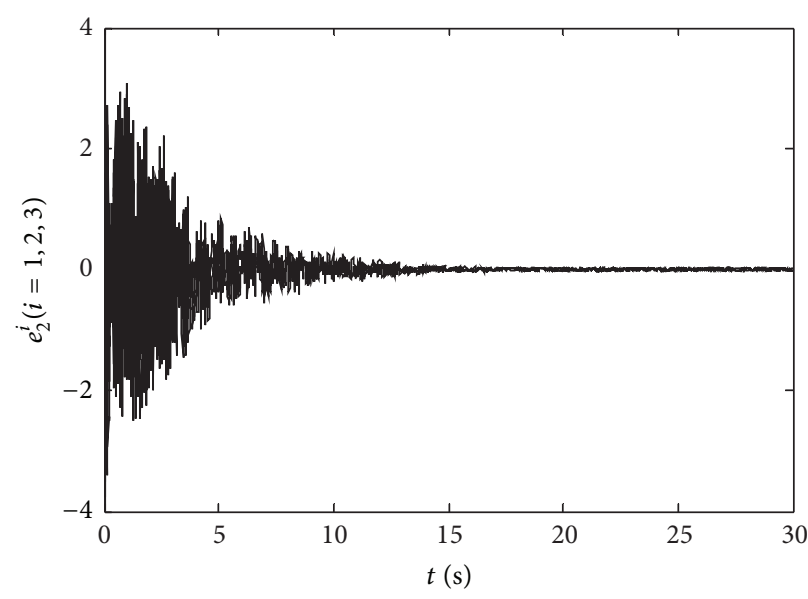

(b)

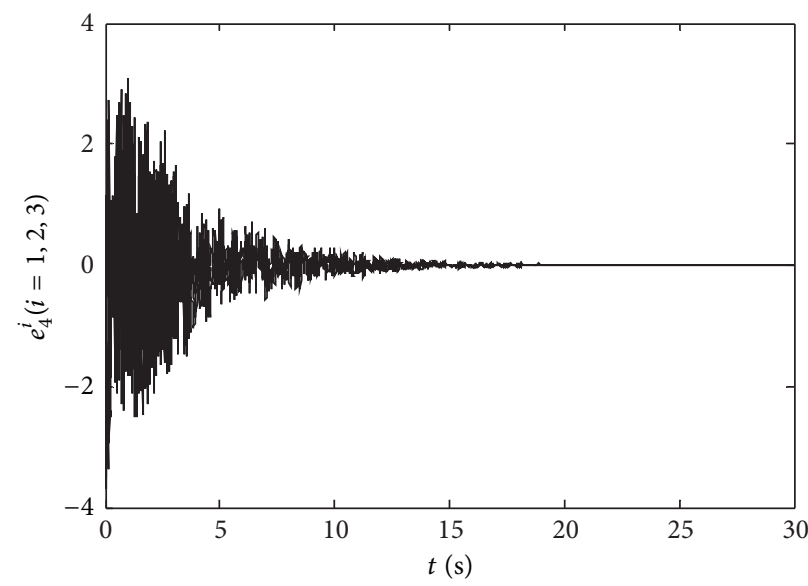

(d)

FIGURE 3: The control impact on the convergence errors of network outer synchronization.

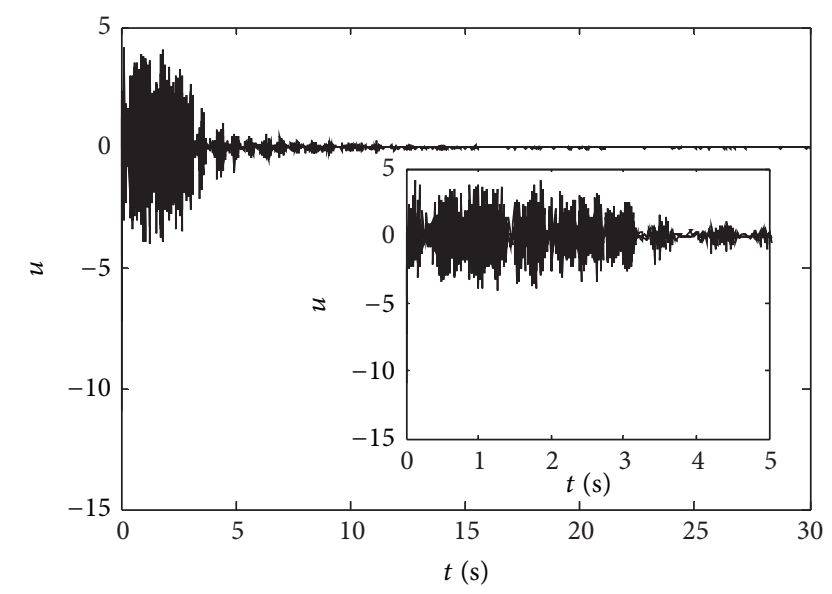

FIGURE 4: The convergence process with the proposed controller design.

\section{Acknowledgments}

This work is supported in part by the National High Technology Research and Development Program of China (Grant no.
2012AA051704) and National Natural Science Foundation of China (Grant no. 51107113).

\section{References}

[1] J. Feng, S. Q. Wang, and Z. S. Wang, "Stochastic synchronization in an array of neural networks with hybrid nonlinear coupling," Neurocomputing, vol. 74, no. 18, pp. 3808-3815, 2011.

[2] Y. H. Xu, W. N. Zhou, J. A. Fang, and H. Q. Lu, "Structure identification and adaptive synchronization of uncertain general complex dynamical networks," Physics Letters A: General, Atomic and Solid State Physics, vol. 374, no. 2, pp. 272-278, 2009.

[3] B. X. Wang and Z.-H. Guan, "Chaos synchronization in general complex dynamical networks with coupling delays," Nonlinear Analysis: Real World Applications, vol. 11, no. 3, pp. 1925-1932, 2010.

[4] Z. Y. Wu and X. C. Fu, "Outer synchronization between driveresponse networks with nonidentical nodes and unknown parameters," Nonlinear Dynamics, vol. 69, no. 1-2, pp. 685-692, 2012.

[5] W. Sun, Z.-Z. Yan, S.-H. Chen, and J.-H. Lü, "Outer synchronization of complex networks by impulse," Communications in Theoretical Physics, vol. 56, no. 5, pp. 885-890, 2011. 
[6] J.-W. Wang, Q. H. Ma, L. Zeng, and M. S. abd-Elouahab, "Mixed outer synchronization of coupled complex networks with timevarying coupling delay," Chaos, vol. 21, no. 1, Article ID 013121, 2011.

[7] C. P. Li, W. G. Sun, and J. G. Kurths, "Synchronization between two coupled complex networks," Physical Review E-Statistical, Nonlinear, and Soft Matter Physics, vol. 76, no. 4, Article ID 046204, 6 pages, 2007.

[8] M. Sun, C. Y. Zeng, and L. X. Tian, "Linear generalized synchronization between two complex networks," Communications in Nonlinear Science and Numerical Simulation, vol. 15, no. 8, pp. 2162-2167, 2010.

[9] Z. Q. Yang, Q. Zhang, and Z. Q. Chen, "Adaptive linear generalized synchronization between two nonidentical networks," Communications in Nonlinear Science and Numerical Simulation, vol. 17, no. 6, pp. 2628-2636, 2012.

[10] G. J. Wang, J. D. Cao, and J. Q. Lu, "Outer synchronization between two nonidentical networks with circumstance noise," Physica A: Statistical Mechanics and Its Applications, vol. 389, no. 7, pp. 1480-1488, 2010.

[11] C.-X. Fan, G.-P. Jiang, and F.-H. Jiang, "Synchronization between two complex dynamical networks using scalar signals under pinning control," IEEE Transactions on Circuits and Systems I: Regular Papers, vol. 57, no. 11, pp. 2991-2998, 2010.

[12] M. M. Asheghan, J. Míguez, M. T. Hamidi-Beheshti, and M. S. Tavazoei, "Robust outer synchronization between two complex networks with fractional order dynamics," Chaos, vol. 21, no. 3, Article ID 033121, 2011.

[13] Y. Q. Wu, C. P. Li, Y. J. Wu, and J. Kurths, "Generalized synchronization between two different complex networks," Communications in Nonlinear Science and Numerical Simulation, vol. 17, no. 1, pp. 349-355, 2012.

[14] S. Zheng, S. G. Wang, G. G. Dong, and Q. S. Bi, "Adaptive synchronization of two nonlinearly coupled complex dynamical networks with delayed coupling," Communications in Nonlinear Science and Numerical Simulation, vol. 17, no. 1, pp. 284-291, 2012.

[15] J. Mei, M. H. Jiang, and Z. C. Huang, "Outer synchronization between two complex networks with identical and nonidentical topological structures," in Proceedings of the 4th International Workshop on Advanced Computational Intelligence (IWACI '11), pp. 757-762, Hubei, China, October 2011.

[16] X. J. Wu and H. T. Lu, "Hybrid synchronization of the general delayed and non-delayed complex dynamical networks via pinning control," Neurocomputing, vol. 89, pp. 168-177, 2012.

[17] W. L. Guo, "Lag synchronization of complex networks via pinning control," Nonlinear Analysis: Real World Applications, vol. 12, no. 5, pp. 2579-2585, 2011.

[18] X. J. Wu and H. T. Lu, "Outer synchronization of uncertain general complex delayed networks with adaptive coupling," Neurocomputing, vol. 82, pp. 157-166, 2012.

[19] X. J. Wu and H. T. Lu, "Generalized function projective (lag, anticipated and complete) synchronization between two different complex networks with nonidentical nodes," Communications in Nonlinear Science and Numerical Simulation, vol. 17, no. 7, pp. 3005-3021, 2012.

[20] Q. J. Zhang and J. C. Zhao, "Projective and lag synchronization between general complex networks via impulsive control," Nonlinear Dynamics, vol. 67, no. 4, pp. 2519-2525, 2012.

[21] M. F. Hu, Z. Y. Xu, and Y. Q. Yang, "Projective cluster synchronization in drive-response dynamical networks," Physica A:
Statistical Mechanics and Its Applications, vol. 387, no. 14, pp. 3759-3768, 2008.

[22] X. J. Wu and H. T. Lu, "Projective lag synchronization of the general complex dynamical networks with distinct nodes," Communications in Nonlinear Science and Numerical Simulation, vol. 17, no. 11, pp. 4417-4429, 2012.

[23] M. F. Hu, Y. Q. Yang, Z. Y. Xu, R. Zhang, and L. X. Guo, "Projective synchronization in drive-response dynamical networks," Physica A: Statistical Mechanics and Its Applications, vol. 381, no. 1-2, pp. 457-466, 2007.

[24] J. R. Chen, L. C. Jiao, J. S. Wu, and X. D. Wang, "Projective synchronization with different scale factors in a drivenresponse complex network and its application in image encryption," Nonlinear Analysis: Real World Applications, vol. 11, no. 4, pp. 3045-3058, 2010.

[25] L. H. Huang, Z. Y. Wang, Y. N. Wang, and Y. Zuo, "Synchronization analysis of delayed complex networks via adaptive timevarying coupling strengths," Physics Letters A: General, Atomic and Solid State Physics, vol. 373, no. 43, pp. 3952-3958, 2009.

[26] Z. Li, L. C. Jiao, and J.-J. Lee, "Robust adaptive global synchronization of complex dynamical networks by adjusting timevarying coupling strength," Physica A: Statistical Mechanics and Its Applications, vol. 387, no. 5-6, pp. 1369-1380, 2008.

[27] P. Li and Z. Yi, "Synchronization analysis of delayed complex networks with time-varying couplings," Physica A: Statistical Mechanics and Its Applications, vol. 387, no. 14, pp. 3729-3737, 2008.

[28] P. deLellis, M. diBernardo, and F. Garofalo, "Novel decentralized adaptive strategies for the synchronization of complex networks," Automatica, vol. 45, no. 5, pp. 1312-1318, 2009.

[29] J. H. Koo, D. H. Ji, and S. C. Won, "Synchronization of singular complex dynamical networks with time-varying delays," Applied Mathematics and Computation, vol. 217, no. 8, pp. 39163923, 2010.

[30] D. H. Ji, J. H. Park, W. J. Yoo, S. C. Won, and S. M. Lee, "Synchronization criterion for Lur'e type complex dynamical networks with time-varying delay," Physics Letters A: General, Atomic and Solid State Physics, vol. 374, no. 10, pp. 1218-1227, 2010.

[31] B. Liu, W. Lu, and T. P. Chen, "Synchronization in complex networks with stochastically switching coupling structures," IEEE Transactions on Automatic Control, vol. 57, no. 3, pp. 754760, 2012.

[32] X. Zhou, H. Feng, and S. H. Chen, "The effect of control strength on the synchronization in pinning control questions," Computers and Mathematics with Applications, vol. 61, no. 8, pp. 2014-2018, 2011.

[33] T. H. Lee, J. H. Park, D. H. Ji, O. M. Kwon, and S. M. Lee, "Guaranteed cost synchronization of a complex dynamical network via dynamic feedback control," Applied Mathematics and Computation, vol. 218, no. 11, pp. 6469-6481, 2012. 


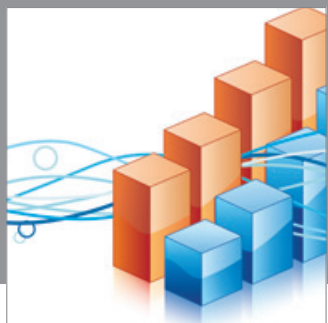

Advances in

Operations Research

mansans

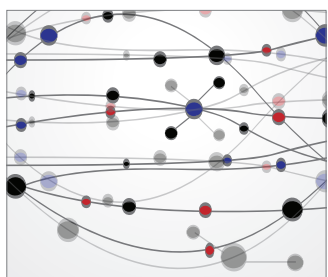

The Scientific World Journal
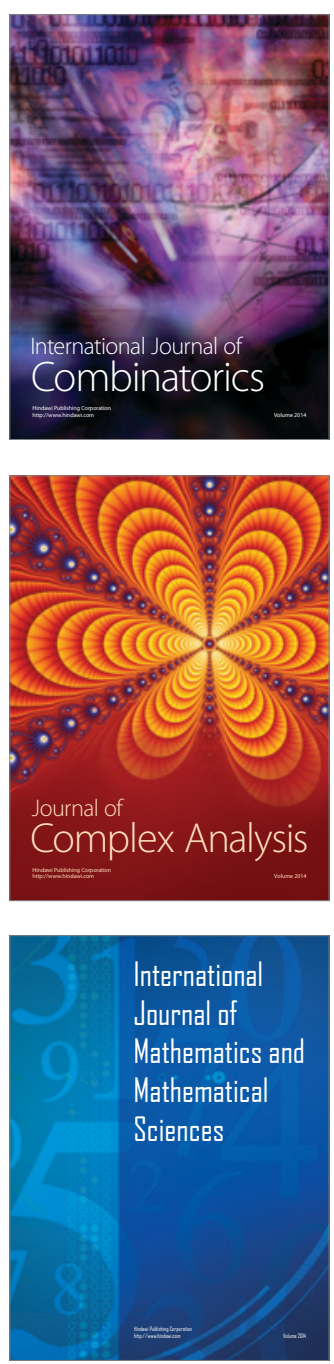
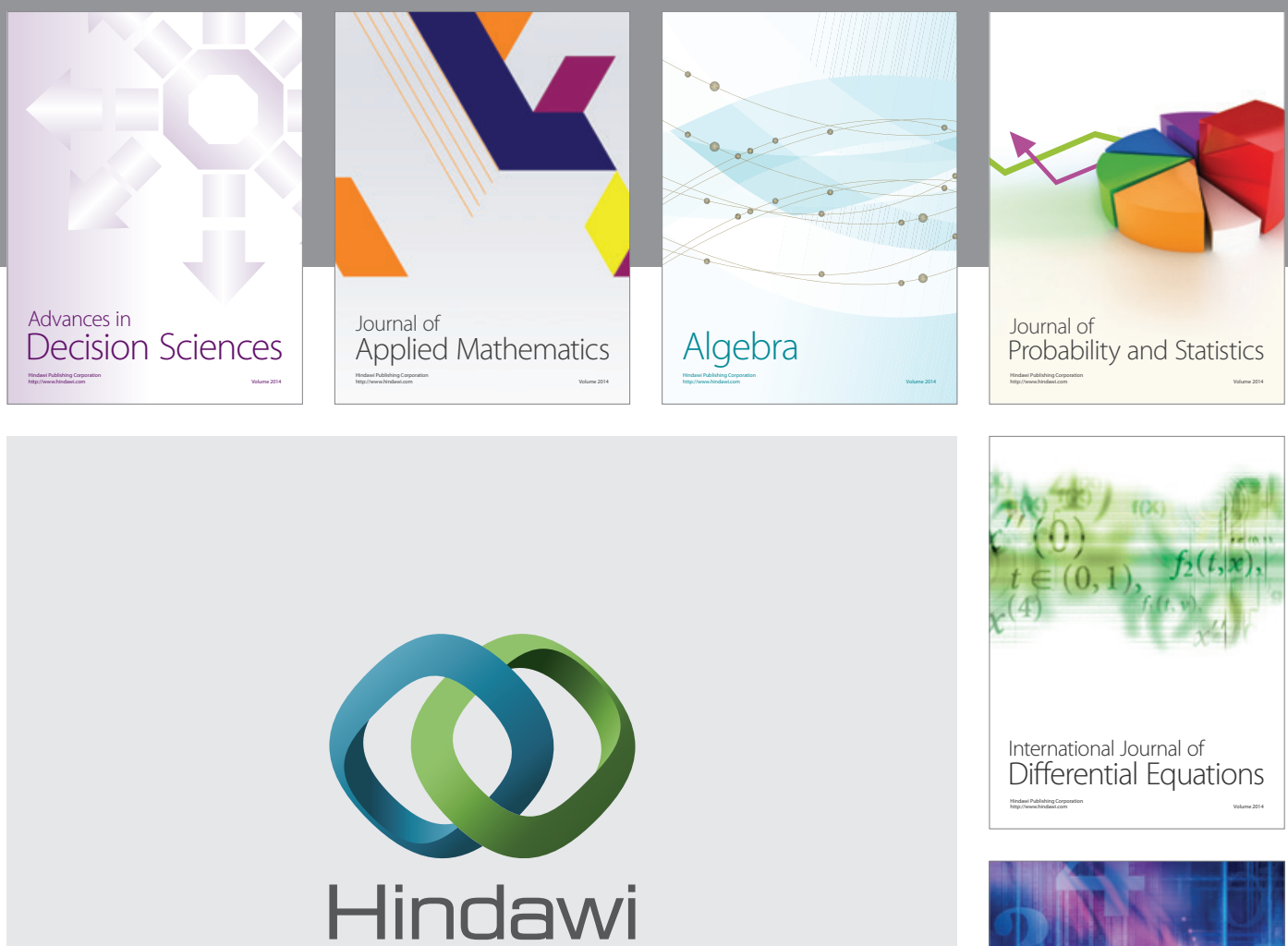

Submit your manuscripts at http://www.hindawi.com
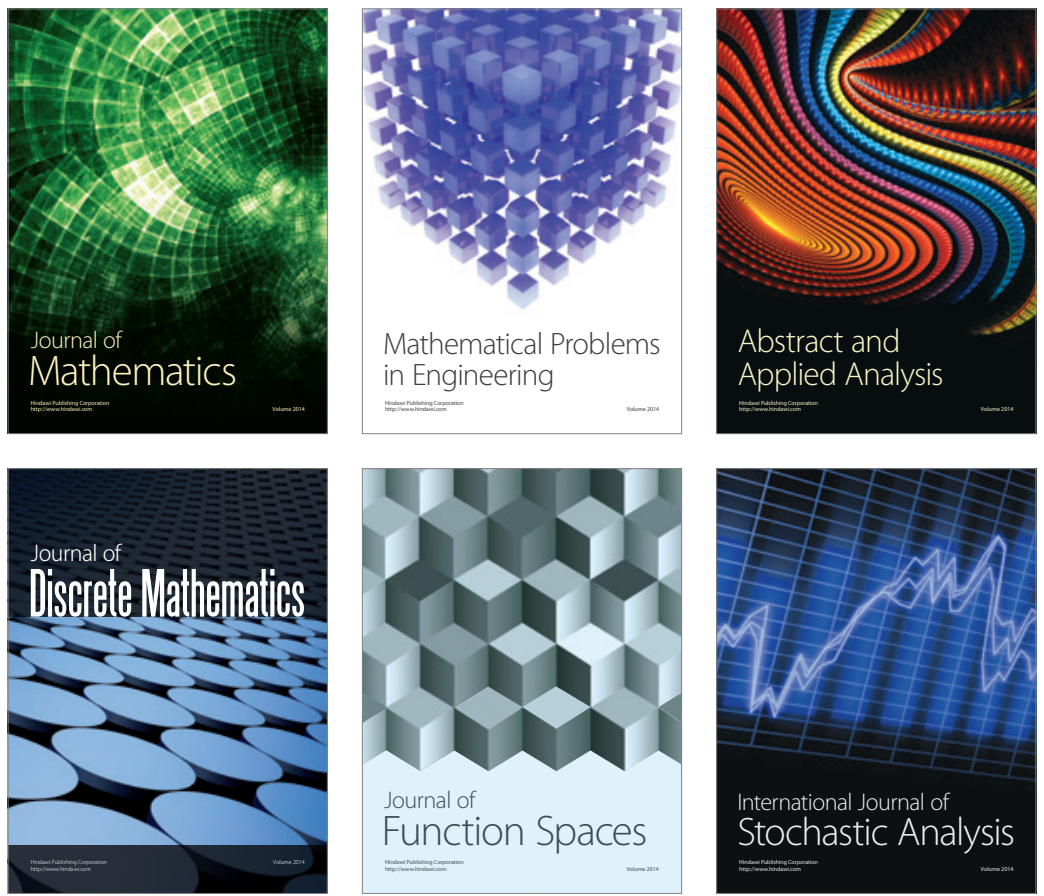

Journal of

Function Spaces

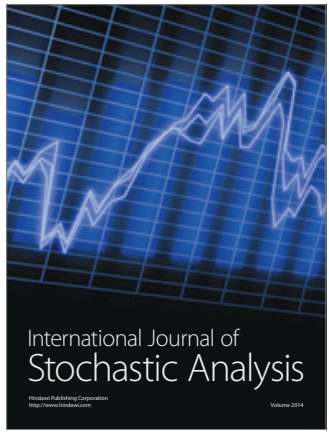

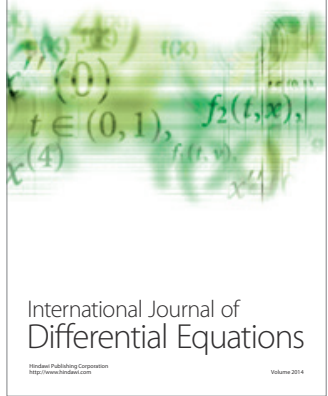
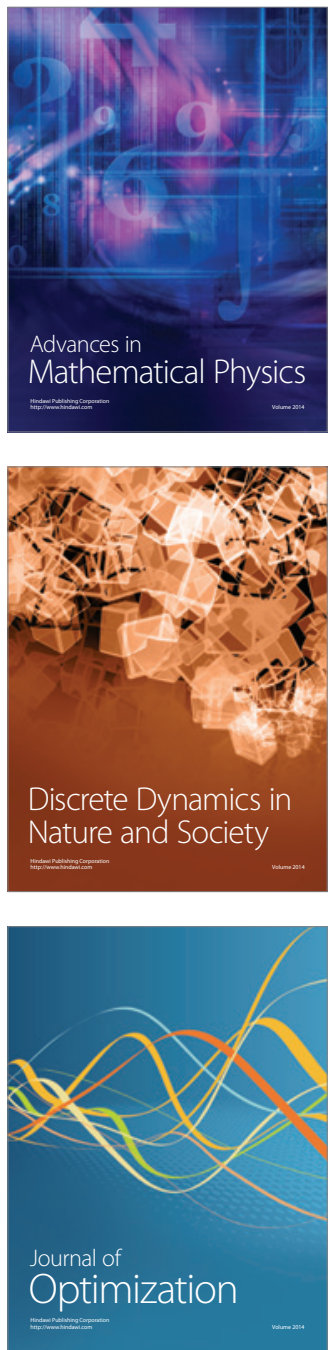